\title{
Utilidad de un nuevo sistema de cronometraje para proporcionar feedback inmediato a los nadadores
}

S. LLANA, P. PERÉZ.

Departamento de Educación Física y Deportiva. Universitat de València.

\begin{abstract}
Resumen
El presente documento tiene dos objetivos, en primer lugar, describir un nuevo sistema de cronometraje que proporciona feedback en tiempo real y sin interferir en la ejecución del nadador y, en segundo lugar, comprobar su utilidad. El sistema consiste en una pantalla de leds, resistente al agua e instalada en el fondo de la piscina, conectada a una Unidad Central mediante una plataforma de contacto colocada en la pared donde se realiza el viraje. La pantalla de leds informa al nadador de los tiempos y número de vueltas e, incluso, puede presentar texto (consignas) escrito por el entrenador. Este sistema se puede conectar a un PC o una PDA, permitiendo registrar los tiempos para su posterior análisis. Se comparó la eficacia del feedback proporcionado por este sistema, con la condición «feedback tradicional» y la condición «sin feedback», a ritmo de nado aeróbico. Los resultados muestran que la dispersión de los tiempos parciales los nadadores, es menor con el feedback proporcionado por el nuevo sistema.
\end{abstract}

Palabras clave: Feedback, natación, biomecánica.

\section{Introducción}

Tanto en el proceso de aprendizaje, como en el de perfeccionamiento de la técnica de nado, la retroinformación (feedback) que el nadador recibe sobre su ejecución técnica juega un papel clave para la mejora-consolidación del gesto técnico (Bilodeau, 1966; Deligneres, 2005). El feedback intrínseco al nadador está siempre presente, pues éste proviene de la información que el sistema nervioso recibe desde los diferentes receptores del cuerpo humano (Latash, 1998): propioceptores que proporcionan información muscular y articular, exteroceptores que proporcionan información de la piel (mucho más importante en natación que en los deportes terrestres) y los receptores vestibulares, que proporcionan información

Correspondencia:

Dpto. de Educación Física y Deportiva.

Universitat de València.

Dr. Salvador Llana Benlloch

E-mail: salvador.llana@uv.es / Telf. 649989424

Dr. Pedro Pérez Soriano

E-mail: pedro.perez-soriano@uv.es referente a la orientación del cuerpo en el espacio. En la jerga del entrenamiento diario, el feedback intrínseco se incluye dentro de las denominadas «sensaciones» del nadador.

El feedback extrínseco al nadador depende de que «algo» 0 «alguien» le proporcione información sobre su ejecución técnica. Dado que «las sensaciones» del nadador con respecto a su nivel de ejecución técnica no siempre coinciden con la apreciación externa, los expertos (Hay \& Reid, 1988) coinciden en la importancia que tiene el feedback externo en la fase de detección de errores. Pero, además de informar al nadador sobre el resultado de su gesto motor, el feedback extrínseco lleva implícitas otras funciones como (Newell, 1976): función de guía del aprendizaje, función asociativa y, por último, función motivadora o de incentivo. Estas funciones no son excluyentes, sino que pueden estar presentes al mismo tiempo, de esta manera se aumenta la probabilidad de que el siguiente ensayo se oriente en la dirección adecuada.

El tipo de feedback extrínseco tradicional es el que proporciona el entrenador a pie de piscina. Sin embargo, tal y como los avances tecnológicos lo 
van permitiendo, cada vez se utilizan más instrumentos para recabar información válida, precisa y fiable, encaminada a orientar, de forma cada vez más eficiente, el aprendizaje de los nadadores (Pérez y cols, 2005).

En este sentido, la natación es una de las modalidades deportivas que mayor atención ha prestado, desde la década de 1960, a la aplicación de avances tecnológicos encaminados a mejorar las diferentes facetas del proceso de entrenamiento. Estas tecnologías permiten cuantificar la calidad técnica de los deportistas, crear modelos técnicos o patrones y establecer comparaciones con ellos mismos y/o con otros deportistas, y en distintos periodos de la temporada (Brizuela y Llana, 1997). De esta manera, se presenta un feedback extrínseco de gran utilidad para nadadores y entrenadores. Sin embargo, este feedback no suele ser inmediato, proporcionándose días, o en el mejor de los casos horas, después del entrenamiento. No obstante, tal y como avanza la tecnología este periodo de tiempo también se acorta, por ejemplo, en las décadas de 1980 y 1990 se utilizaba la fotogrametría-cine (Scheinhauff, 1979) para obtener datos de la biomecánica del nado, lo que suponía varios meses hasta la obtención de los resultados. En la actualidad, se utiliza la fotogrametría-vídeo, que permite obtener los resultados en cuestión de días o, incluso, de horas (Brizuela \& Llana, 1997). Incluso existen sistemas, como el $\mathrm{VICON}^{\circledR}$, que permite obtener los resultados en tiempo real, pero, estos últimos, no se pueden utilizar en el ambiente de las piscinas.

Otro aspecto que ha demandado gran interés en los últimos años, es el de mejorar la comunicación entrenador-nadador durante el nado (Pérez y cols, 2004). Esto es así, porque el medio acuático dificulta en gran medida que el entrenador proporcione información referente a los tiempos de paso, metros nadados o ejecución técnica durante el nado. Los entrenadores deben utilizar señales visuales desde fuera de la piscina que no siempre son advertidas por los nadadores. Ello requiere que entrenador y nadador dediquen parte de su atención a estar pendientes de dichas señales, pudiendo repercutir negativamente en su atención de otros aspectos de la tarea.

Esto es de gran importancia, pues la falta de comunicación y de información en tiempo real, perjudica el aprendizaje del deportista, dado que es en esta fase del entrenamiento y ese tipo de información la mas eficaz respecto al aprendizaje y la mejora de la técnica deportiva (Del Villar y Fuentes, 1999).
Con el propósito de mejorar dicha situación, se han desarrollado sistemas de luces subacuáticas que marcan el ritmo de nado, liberando mentalmente al nadador y permitiendo al entrenador fijar su atención en otros aspectos (González y cols. 2002). Sin embargo, este sistema no soluciona todos los problemas: en primer lugar, porque durante los entrenos no siempre se ha de nadar a ritmos fijos y predeterminados, en segundo lugar, por el tiempo que se pierde en su instalación-desinstalación y, en tercer lugar, porque no proporciona feedback referente a la ejecución técnica.

Otros sistemas desarrollados, como los auriculares acuáticos integrados en un tubo de buceo (e.j. AQUAFM ${ }^{\circledR}$ ) tienen el inconveniente de que interfieren directamente con el nadador, modificando la técnica de ejecución, especialmente en lo virajes, e impidiendo respirar al nadador con normalidad.

Para intentar solucionar parte de la problemática existente en la comunicación entrenador-nadador, se planeó un proyecto cuyos objetivos fueran proporcionar feedback extrínseco al nadador en tiempo real referente, tanto a su ritmo de nado como al número de vueltas. Todo ello, basado en una pantalla subacuática con leds que pudiera ver el nadador tras cada viraje.

\section{Materiales y métodos}

Fase 1: Diseño, construcción y verificación del sistema de cronometraje

Para lograr los objetivos arriba expuestos, se diseñó el sistema expuesto en la figura 1.

El nuevo sistema de cronometraje acuático esta formado por dos elementos claramente diferenciados: Hardware (estructura o soporte físico) y software (programas e instrucciones informáticas).

\section{* Hardware:}

Se encuentra dividido en dos unidades, la unidad central y accesorios, que quedan fuera del agua, y la pantalla de leds que se deposita al fondo de la piscina:

- Unidad central, botonera de inicio/parada y transductor telemétrico: El sistema de cronometraje está gobernado por un microcontrolador (AT89C52) que representa la unidad central del cronómetro. Éste almacena los entrenamientos en una memoria E2PROM (24LC64) capaz de almacenar un número determinado de sesiones de entrenamiento. Esta unidad permite la adquisición de las señales que identifican los tiempos del nadador. 
La puesta en marcha y apagado del sistema se realiza mediante la botonera de inicio/parada que se encuentra conectada a la unidad central. Una vez puesto en marcha el sistema, los tiempos parciales son enviados al display subacuático mediante una transmisión serie asíncrona de los datos obtenidos. Asimismo, estos datos son enviados a un PC, mediante radiofrecuencia (XTR869), utilizando para ello dos transductores telemétricos.

- Pantalla de leds subacuática: Es el letrero luminoso colocado en el fondo de la piscina. Gestiona los datos recibidos de la unidad de control, de manera que, tras cada viraje, le muestra al nadador los tiempos parcial y total, así como el número de vueltas realizadas. La información que presenta el sistema al nadador consiste en: tiempo total (leds verdes), tiempos parciales (leds rojos) y de numero de vueltas (leds verdes de menor dimensión),

Todo este soporte físico está alimentado por dos fuentes de energía. La primera de ellas es una batería de $12 \mathrm{v}$ y $10 \mathrm{Ah}$, encargada de alimentar la unidad de control, letrero luminoso, botonera y sensor final de carrera, regulando de un modo adecuado el voltaje (entre 12 v y 5 v) que necesita cada una de estas partes. La segunda fuente de energía es la propia del PC, la cual, a través del puerto USB suministra la corriente necesaria para alimentar la circuitería conectada como receptor de radiofrecuencia.

\section{* Software:}

Es el soporte lógico o conjunto de programas e instrucciones que dirige el funcionamiento del sistema. Está formulado en lenguaje $\mathrm{C}$ de programación. Gracias a éste, los microcontroladores empleados permiten realizar tareas secuenciales previamente diseñadas, controlando el cronometraje, comunicaciones, representación en pantalla de dígitos, etc.

Además, y para su mejor gestión en el PC, se ha implementado mediante la herramienta de

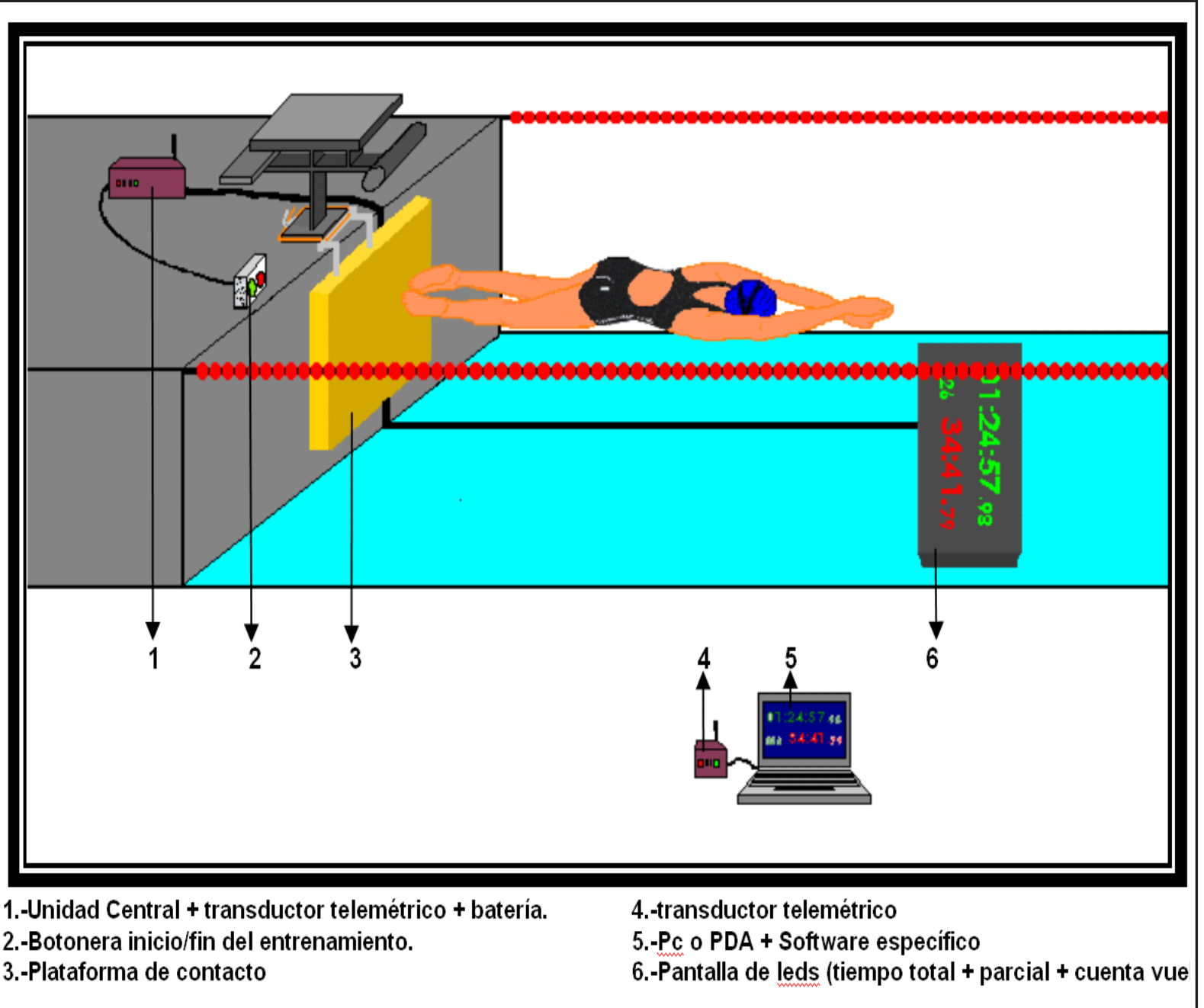

Figura 1. Esquema general del sistema 
programación Visual Basic, un programa de ventanas que permite trabajar en el sistema operativo Windows.

El nadador acciona el cronómetro interno del sistema mediante una placa de contacto portátil de fácil instalación. Dicho sistema es adaptable a cualquier piscina que disponga de poyete, mediante un anclaje de acero inoxidable, que mantiene la plataforma de contacto en el lugar deseado por el entrenador. A su vez el conjunto compuesto por la placa de contacto y el anclaje, se sujeta al poyete mediante un cinturón de seguridad de alta resistencia.

El sistema al completo es transportable en un pequeño carro, donde se disponen todos los componentes del instrumento, pudiendo manejar el conjunto del sistema una sola persona.

\section{Fase 2: Pruebas de feedback}

En el estudio participaron de forma voluntaria 6 nadadores de nivel nacional (edad $=16^{\prime} 48 \pm 1^{\prime} 10$ años, altura $=1^{\prime} 756 \pm 0$ 0'084 $\mathrm{m}$, peso $=71^{\prime} 29 \pm 3^{\prime} 51$ $\mathrm{kg})$. Todos firmaron un Manifiesto de Consentimiento para participar en el estudio.

Las pruebas se realizaron en una piscina de 25 m. El entrenador del club seleccionó, de forma individual para cada nadador, una velocidad de nado de carácter aeróbico. Los deportistas nadaron 200 $\mathrm{m}$, de forma randomizada, en tres condiciones diferentes de feedback:

- $\quad$ Sin feedback: el nadador es informado del ritmo antes de iniciar el nado y, durante éste, no se proporciona ningún tipo de información referente a si está cumpliendo con el ritmo previsto. a)

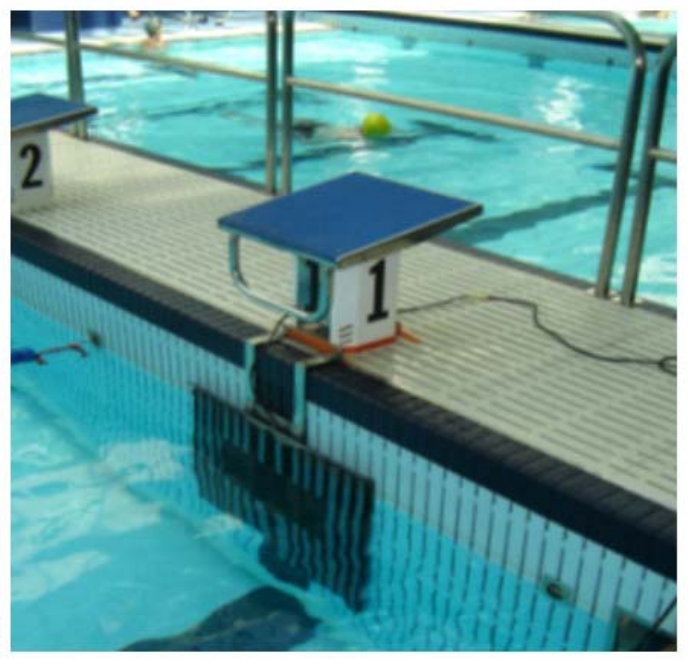

c)

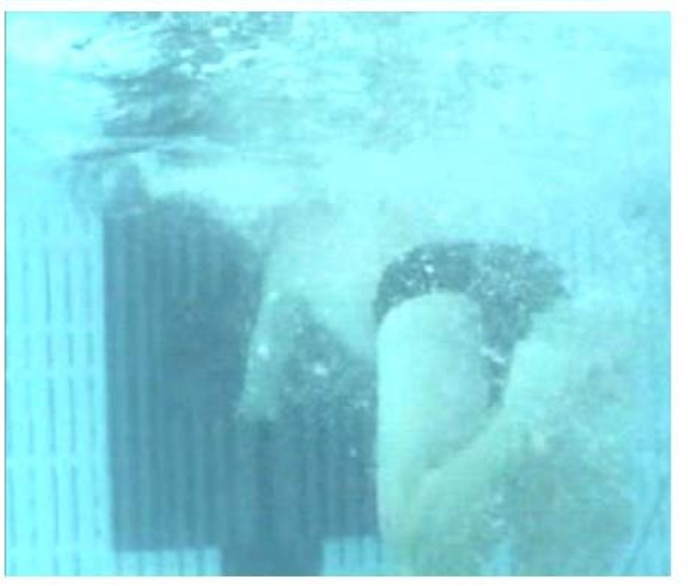

b)

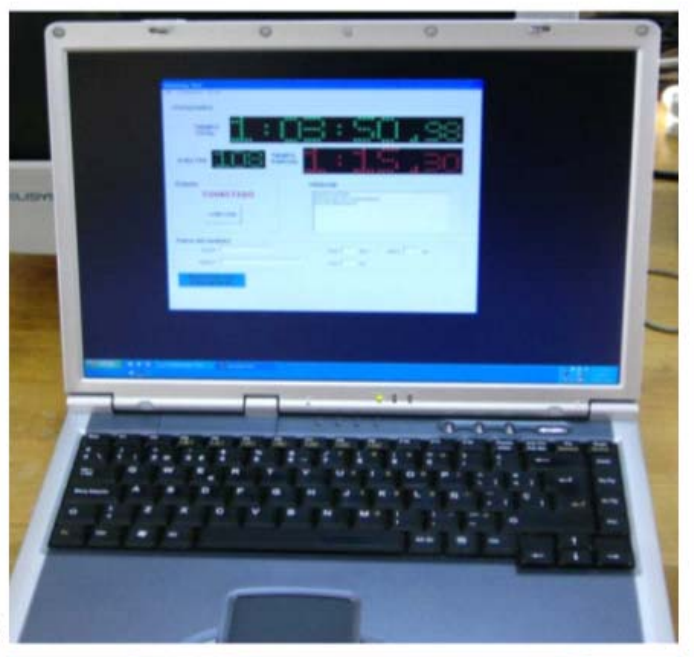

d)

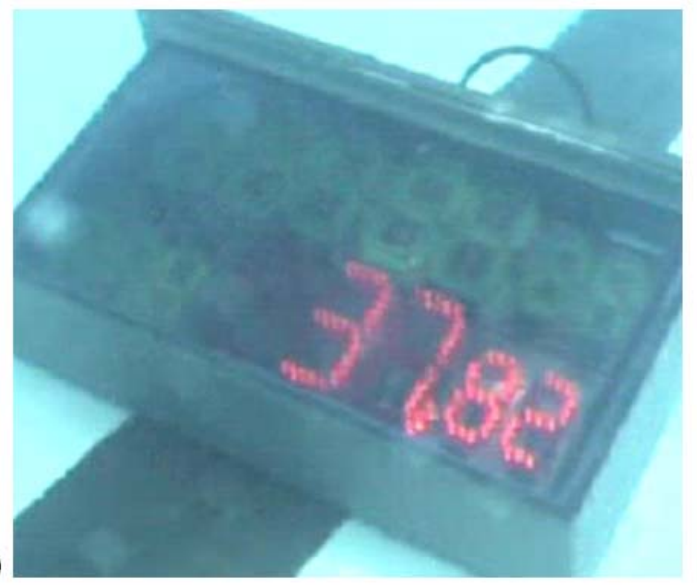

Figura 2. a) sistema anclado al poyete de salida, c) nadador realizando un viraje de braza en la placa de contacto, b) visualización de los tiempos y número de vueltas en un $\mathrm{PC}, \mathrm{y}$ d) pantalla subacuática 
- $\quad$ Con feedback tradicional: el nadador es informado de su ritmo de nado antes de iniciar el nado y, durante éste, recibe información por parte del entrenador que se encuentra a pie de piscina.

- Con feedback proporcionado por el cronómetro acuático: el nadador es informado de su ritmo de nado antes de iniciar el nado y, tras cada viraje, observará sus tiempos parciales en el cronómetro que estará en el fondo de la piscina.

Los tiempos parciales fueron introducidos en el programa Statgraphics v.4.0, para realizar el tratamiento estadístico que, dado el reducido número de la muestra, consistió en un análisis descriptivo de la dispersión (varianza y rango) de los tiempos parciales.

\section{Resultados}

La tabla 1 muestra los resultados para la varianza y para el rango obtenidos en las tres condiciones de feedback, y a una velocidad de nado de carácter aeróbico.

\section{Discusión}

Los resultados obtenidos muestran una menor dispersión en los tiempos parciales de los nadadores con el nuevo sistema de cronometraje, que con las otras dos condiciones de feedback examinadas ( $\sin$ feedback y feedback tradicional).

Estos resultados coinciden con los establecidos por investigadores de aprendizaje motor (Del Villar y Fuentes, 1999), según los cuales, el feedback más efectivo es el que se proporciona en tiempo real y sin interferir con la ejecución técnica de los nadadores.

No obstante, la muestra analizada ha sido reducida (6 nadadores) y que sólo se ha probado a un ritmo de nado (ritmo de «aeróbico ligero» en terminología de Entrenamiento Deportivo). Por ello, para verificar la utilidad del nuevo sistema de cronometraje, hay que aumentar la muestra utilizada, así como probarlo en diferentes ritmos de nadado, tanto aeróbicos como anaeróbicos.

\begin{tabular}{|l|l|l|l|}
\hline & Sin feedback & Feedback tradicional & Nuevo sistema \\
\hline Varianza & $2^{\prime} 3382 \mathrm{~s}$ & $2^{\prime} 1154 \mathrm{~s}$ & $0^{\prime} 9756 \mathrm{~s}$ \\
\hline Rango & $4^{\prime} 92 \mathrm{~s}$ & $5^{\prime} 0 \mathrm{~s}$ & $3^{\prime} 23 \mathrm{~s}$ \\
\hline
\end{tabular}

Tabla 1. 


\section{Agradecimientos}

Este trabajo se ha podido realizar gracias a una beca del Consejo Superior de Deportes en el año 2004. Proyecto 06/UPR10/04.

\section{Bibliografía}

1. Bilodeau, I. M. (1966). Information feedback. In E. A. Bilodeau (Ed.). Adquisition of skill (Vol. 55, pp. 603-612). New York: Academic Press.

2. Brizuela, G; Llana, S. (1997). Herramientas y técnicas para el análisis biomecánico. En Camarero, S; Tello, V; Mundana, J. (1997). Análisis de la práctica deportiva. Valencia: Promolibro.

3. Deligneres, D. (2005) Learning complex motro coordinations, a co-operation between spontaneus ans expert co-ordination. First Internationls Symposium on Swimming, Paris.

4. Del Villar, F. y Fuentes, J. P. (1999). Las destrezas docentes en la enseñanza de tenis. En J. P. Fuentes, E. M. Cervelló, F. Del Villar, N. Gusi y F. J. Moreno, Enseñanza y entrenamiento del tenis. Fundamentos didácticos y científicos (pp. 1-36). Cáceres: Universidad de Extremadura, Servicio de Publicaciones.
5. Gonzalez, V; Sanchís, E; Villalobos, M; Brizuela, G; Llana, S; Tella, V. (2002). A new electronic system for the control of swimming speed. IX World Symposium of Biomechanics and Medicine in Swimming. 21-23 june, Saint-Etienne. France.

6. Hay, J. \& Reid, J. (1988) Anatomy, Mechanics and Human Motion. Ed. Prentice-Hall.

7. Latash, M. (1998) Neurophysiological Basis of Movement. Human Kinetics

8. Martens, R. (1987). Coavhes quide to sport psychology. Ed. Human Kinetics.

9. Newell, K. M. (1976). Knowledge of results and motor learning. In J. Keogh \& R. S. Hutton (Eds.). Exercise and Sports Sciences Reviews. Santa Barbara, California: Journal Publishing Affiliats.

10. Pérez. P; Llana, S; Zahonero, J. y García, E. (2004) Sistema de cronometraje con feedback en tiempo real para el nadador. XXIV Congreso Internacional de Técnicos de Natación. Huesca.

12. Pérez, P; Llana, S; Zahonero, J. y Navarro, P. (2005) A new chronometer system to provide real time feedback to swimmers. First Internationls Symposium on Swimming, Paris.

13. Scheinhauff, R.E. (1979) A hidrodinamic analysis os swimming propulsión. Internarional Series on Sport Sciences: Swimming III (Ed. Terauds \& Bedingfield). Baltimore: Universitry Park Press. 